\title{
Árvores Ramsey-restritas mínimas
}

\author{
Maurício Collares ${ }^{1}$, Antônio K. B. Fernandes ${ }^{1}$, Guilherme O. Mota $^{2}$, Hugo M. Vicente ${ }^{1 *}$ \\ ${ }^{1}$ Instituto de Ciências Exatas - Universidade Federal de Minas Gerais (UFMG) \\ ${ }^{2}$ Instituto de Matemática e Estatística - Universidade de São Paulo (USP) \\ mauricio@collares.org, antoniokaique@hotmail.com \\ motalime.usp.br, hugovicentedyahoo.com.br
}

\begin{abstract}
Given graphs $G, S$ and $H$, we write $G \stackrel{m r}{\longrightarrow}(S, H)$ if every edgecolouring of $G$ contains a monochromatic copy of $S$ or a rainbow copy of $H$. We prove that, for $S=K_{1,3}$ and $H$ a complete binary tree of height $h$, the smallest tree $T$ satisfying $T \stackrel{m r}{\longrightarrow}(S, H)$ has size $2^{(1 / 2+o(1)) h^{2}}$.

Resumo. Para grafos $G, S$ e $H$, dizemos que $G \stackrel{m r}{\longrightarrow}(S, H)$ se toda coloração das arestas de $G$ tem uma cópia monocromática de $S$ ou uma cópia multicolorida de $H$. Provamos que se $S=K_{1,3}$ e $H$ é uma árvore binária completa de altura h, então o tamanho da menor árvore $T$ que satisfaz $T \stackrel{m r}{\longrightarrow}(S, H)$ é $2^{(1 / 2+o(1)) h^{2}}$.
\end{abstract}

\section{Introdução}

Para grafos $G, S$ e $H$ dizemos que uma coloração de $E(G)$ é $(S, H)$-restrita se ela não contém uma cópia monocromática de $S$ nem uma cópia multicolorida de $H$ (uma cópia de $H$ em que todas as arestas têm cores diferentes). Denotaremos a propriedade " $G$ não admite coloração $(S, H)$-restrita" por $G \stackrel{m r}{\longrightarrow}(S, H)$. O número de Ramsey restrito $r_{c}(S, H)$, definido como o menor $n$ tal que $K_{n} \stackrel{m r}{\longrightarrow}(S, H)$, foi estudado por diversos autores (veja, e.g., [Alon et al. 2003, Gyárfás et al. 2007, Loh and Sudakov 2009]).

Em [Jamison et al. 2003] é mostrado que o $r_{c}(S, H)$ existe se e somente se $S$ é uma estrela ou $H$ é uma floresta. Em [Collares et al. 2021], foi determinado o limiar para a propriedade $G(n, p) \stackrel{m r}{\longrightarrow}(S, H)$ no caso em que $H$ é uma floresta, onde $G(n, p)$ denota o grafo aleatório de Erdős-Rényi. Em particular, no caso em que $S$ é uma estrela, o limiar depende do parâmetro

$$
m_{F}(S, H)=\min \{k(G) \mid G \text { é floresta e } G \stackrel{m r}{\longrightarrow}(S, H)\}
$$

onde $k(G)$ denota o tamanho da maior componente conexa de $G$. Neste artigo iremos investigar a ordem de crescimento do parâmetro $m_{F}(S, H)$ quando $S=K_{1,3}$, a estrela de três pontas, em termos do tamanho de $H$. Quando $H$ é um caminho com $d$ arestas (denotado por $P_{d}$ ), mostramos que $m_{F}\left(K_{1,3}, H\right)=d^{(1 / 2+o(1)) d}$. Quando $H$ é uma árvore binária completa de altura $h$ (denotada por $T_{h}$ ), mostramos que $m_{F}\left(K_{1,3}, H\right)=2^{(1 / 2+o(1)) h^{2}}$. Tais resultados implicam cotas inferiores e superiores no caso geral.

${ }^{*}$ Guilherme O. Mota teve apoio financeiro de CNPq (304733/2017-2, 428385/2018-4) e FAPESP (2018/04876-1, 2019/13364-7). O presente trabalho foi realizado com apoio da Coordenação de Aperfeiçoamento de Pessoal de Nível Superior - Brasil (CAPES) - Código de Financiamento 001. 


\section{Resultado}

O objetivo deste resumo estendido é provar os Teoremas 2.5 e 2.6, que fornecem cotas inferiores e superiores para $m_{F}\left(K_{1,3}, H\right)$ quando $H$ é uma árvore binária completa ou um caminho. Nossas cotas inferiores são consequências de mostrar, nos Lemas 2.3 e 2.4, que a coloração da Definição 2.1 tem boas propriedades.

As seguintes definições serão úteis. Uma árvore enraizada será denotada por um $\operatorname{par}(H, r)$. Dada uma árvore enraizada $T$ e um vértice $v$, um vértice $w$ é descendente de $v$ se o caminho de $v$ a $w$ é aresta-disjunto do caminho de $v$ à raiz. Denotaremos por $h_{T}(v)$ a distância do vértice $v$ à raiz e por $D_{T}(v)$ o número de descendentes de $v$ (incluindo o próprio $v$ ). Um vértice $w$ é filho de outro vértice $v$ se $v w \in E(T)$ e $h_{T}(w)>h_{T}(v)$.

Definição 2.1 (Coloração por peso). Dada uma árvore enraizada $T$, uma coloração por peso de $T$ é uma função $\chi: E(T) \rightarrow \mathbb{N}$ com a seguinte propriedade. Para qualquer vértice $u$, se $v_{1}, \ldots, v_{k}$ são os filhos de $u$, então $\left\{\chi\left(u v_{1}\right), \ldots, \chi\left(u v_{k}\right)\right\}=\{1, \ldots, k\} e$ $\chi\left(u v_{i}\right)<\chi\left(u v_{j}\right)$ para quaisquer $1 \leq i, j \leq k$ tais que $D\left(v_{i}\right)>D\left(v_{j}\right)$.

Para colorir uma árvore $T$ com uma coloração por peso, ordenamos os filhos de cada vértice $u$ por ordem decrescente de número de descendentes (desempatando de modo arbitrário) e colorimos as arestas entre $u$ e seus filhos nessa ordem usando cores de $1 \mathrm{a} k$.

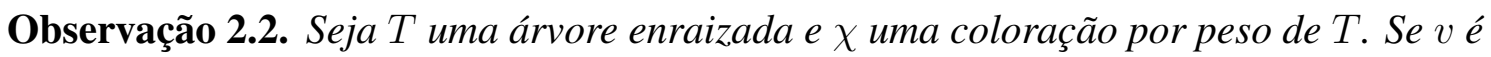
filho de $u$, então $D_{T}(u) \geq \chi(u v) D_{T}(v)$.

Demonstração. $u$ tem pelo menos $\chi(u v)$ filhos com $D_{T}(v)$ ou mais descendentes.

Note que colorações por peso evitam $K_{1,3}$ monocromático. Para estimar o valor $m_{F}\left(K_{1,2}, H\right)$ (que corresponde ao caso de colorações próprias sem cópia multicolorida de $H$ ) em vez de $m_{F}\left(K_{1,3}, H\right)$, a definição de coloração por peso teria que evitar reusar a cor da aresta entre $u$ e seu pai. Assim, na observação acima, o termo $\chi(u v)$ teria que ser substituído por $\max \{1, \chi(u v)-1\}$. É fácil adaptar os argumentos seguintes para tal mudança, mas por simplicidade iremos omitir tais alterações.

A observação acima motiva a seguinte definição. Dada uma árvore não-enraizada $H$ e $s \in V(H)$, defina $\mathcal{P}_{s}(H)$ como o conjunto de todos os caminhos começados em $s$ e terminados numa folha de $H$, e seja

$$
f(H)=\min \left\{\max _{P \in \mathcal{P}_{s}(H)}\left(\prod_{e \in P} \chi(e)\right) \mid \chi: E(H) \rightarrow \mathbb{N} \text { é injetiva e } s \in V(H)\right\} .
$$

Em outras palavras, dada uma coloração multicolorida das arestas de $H$ e um enraizamento $s$ de $H$, consideramos o máximo dos "pesos" (produtório das cores) dos caminhos da raiz até os demais vértices. A conexão entre a função $f$ e cotas inferiores é dada abaixo.

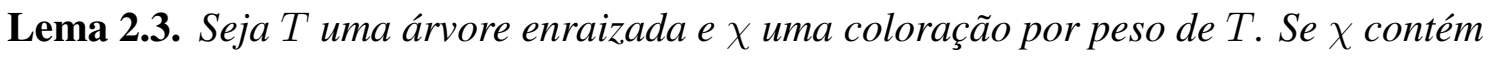
uma cópia multicolorida de $H$, então $|V(T)| \geq f(H)$.

Demonstração. Seja $\varphi: V(H) \rightarrow V(T)$ a imersão de uma cópia multicolorida de $H$ em $T$, e seja $s \in V(H)$ o vértice que minimiza $h_{T}(\varphi(s))$. Temos da definição de $f$ que

$$
\max _{P \in \mathcal{P}_{s}(H)}\left(\prod_{e \in P} \chi(e)\right) \geq f(H) .
$$


Seja $P$ um caminho arbitrário de $\mathcal{P}_{s}(H)$, escreva $P=v_{0} \ldots v_{k}$ e defina $w_{i}=\varphi\left(v_{i}\right)$. Pela escolha de $s, h_{T}\left(w_{0}\right)<\cdots<h_{T}\left(w_{k}\right)$. Aplicando a Observação 2.2 várias vezes, obtemos que $D_{T}(\varphi(s))=D_{T}\left(w_{0}\right) \geq\left(\prod_{i=1}^{k} \chi\left(w_{i-1} w_{i}\right)\right) \cdot D_{T}\left(w_{k}\right)$. Como $P$ era arbitrário e $D_{T}(w) \geq 1$ para todo $w \in T$, temos que

$$
|V(T)| \geq D_{T}(\varphi(s)) \geq \max _{P \in \mathcal{P}_{s}(H)}\left(\prod_{i=1}^{k} \chi\left(w_{i-1} w_{i}\right)\right)
$$

Juntamente com (2), isso conclui a prova do lema.

A árvore binária completa de altura $h$, denotada por $B_{h}$, é a árvore enraizada definida do seguinte modo. A árvore $B_{0}$ é constituída de apenas um vértice, a raiz. Para $h>0$, a árvore $B_{h}$ é a união disjunta de duas cópias $T_{1}$ e $T_{2}$ de $B_{h-1}$ (com raízes $r_{1}$ e $r_{2}$ ), juntamente com um novo vértice $r$ (a nova raiz) que é vizinho de $r_{1}$ e $r_{2}$.

Lema 2.4. Seja $B_{h+1}=(H, r)$ a árvore binária completa de altura $h+1$. Vale que

$$
f(H) \geq 2^{\left(\begin{array}{c}
h \\
2
\end{array}\right) .}
$$

Demonstração. Denote por $T_{1}=\left(H_{1}, r_{1}\right)$ e $T_{2}=\left(H_{2}, r_{2}\right)$ as cópias de $B_{h}$ usadas na construção de $B_{h+1}$. Note que, para qualquer enraizamento $s \in V(H)$, existe $i \in\{1,2\}$ tal que o caminho (em $H$ ) de $s$ a qualquer vértice de $T_{i}$ passa por $r$. Iremos tratar o caso em que $i=1$, pois o outro caso é simétrico. Pela observação anterior, todo $P \in \mathcal{P}^{\prime}:=$ $\mathcal{P}_{r_{1}}\left(H_{1}\right)$ está contido em algum caminho de $\mathcal{P}_{s}(H)$. Assim, por monotonicidade, basta mostrar que, para toda função injetiva $\chi: E\left(T_{1}\right) \rightarrow \mathbb{N}$,

$$
\max _{P \in \mathcal{P}^{\prime}}\left(\prod_{e \in P} \chi(e)\right) \geq 2^{\left(\begin{array}{c}
h \\
2
\end{array}\right)}
$$

Como $T_{1}$ tem $2^{h}$ folhas, vale que $\left|\mathcal{P}^{\prime}\right|=2^{h}$. Usando que o máximo de uma sequência de números é pelo menos a média deles, é suficiente mostrar que

$$
\sum_{P \in \mathcal{P}^{\prime}} \sum_{e \in P} \log _{2} \chi(e) \geq 2^{h}\left(\begin{array}{l}
h \\
2
\end{array}\right) \text {. }
$$

Como $e\left(T_{1}\right)=2^{h+1}-2$, podemos assumir que $\operatorname{Im}(\chi)=\left\{1, \ldots, 2^{h+1}-2\right\}$, pois trocar uma cor por outra de maior valor claramente não diminui o somatório. Seja $E_{i} \subset E\left(T_{1}\right)$ o conjunto das arestas que ligam um vértice de altura $i-1$ a um vértice de altura $i$ em $T_{1}$. Como cada aresta de $E_{i}$ está em $2^{h-i}$ caminhos de $\mathcal{P}^{\prime}$, vale que

$$
\sum_{P \in \mathcal{P}^{\prime}} \sum_{e \in P} \log _{2} \chi(e)=\sum_{i=1}^{h} \sum_{e \in E_{i}} 2^{h-i} \log _{2} \chi(e) .
$$

Aplicando a Desigualdade do Rearranjo, vemos que a soma é minimizada se, e somente se, para todo $1 \leq i<j \leq h$ e qualquer $\operatorname{par}\left(e_{i}, e_{j}\right) \in E_{i} \times E_{j}$, vale que $\chi\left(e_{i}\right)<\chi\left(e_{j}\right)$. 
Como $\left|E_{i}\right|=2^{i}$, uma coloração que minimiza o lado direito de (5) satisfaz $\chi\left(E_{i}\right)=$ $\left\{2^{i}-1, \ldots, 2^{i+1}-2\right\}$. Assim,

$$
\sum_{i=1}^{h} \sum_{e \in E_{i}} 2^{h-i} \log _{2} \chi(e) \geq \sum_{i=1}^{h} 2^{i} \cdot 2^{h-i} \log _{2}\left(2^{i-1}\right)=2^{h} \sum_{i=1}^{h}(i-1)=2^{h}\left(\begin{array}{l}
h \\
2
\end{array}\right) .
$$

Combinando (5) e (6), obtemos (4) e portanto (3), que já argumentamos que implica a desigualdade desejada.

Teorema 2.5. Seja $T_{h}=(H, r)$ a árvore binária completa de altura $h$. Então

$$
m_{F}\left(K_{1,3}, H\right)=2^{(1 / 2+o(1)) h^{2}} .
$$

Demonstração. Começaremos analisando a cota superior. Seja $G$ uma árvore enraizada de altura $h$ em que, para $0 \leq i<h$, os vértices do nível $i$ têm grau $2^{i+3}$. Tal árvore tem $2^{\left(\begin{array}{c}h \\ 2\end{array}\right)+3 h}$ folhas, e portanto $2^{(1 / 2+o(1)) h^{2}}$ vértices. Considere uma coloração de $E(G)$ sem cópia monocromática de $K_{1,3}$. Afirmamos que é possível encontrar uma cópia multicolorida de $T_{h}$ imergindo os vértices "nível a nível". De fato, como cada vértice do nível $i$ de $G$ é incidente a arestas de pelo menos $2^{i+2}$ cores e a restrição de $T_{h}$ aos vértices dos níveis $0, \ldots, i+1$ tem $2^{i+2}-2$ arestas, há uma cor disponível ao imergir um filho de um vértice do nível $i$.

Para provar a cota inferior, relacionaremos os Lemas 2.3 e 2.4. Seja $F$ uma floresta que tem uma cópia multicolorida de $H$ em qualquer coloração que evita $K_{1,3}$. Enraizamos cada componente de $F$ arbitrariamente e as colorimos com uma coloração por peso. Seja $T$ uma componente que contém uma cópia multicolorida de $H$. Pelos Lemas 2.3 e 2.4

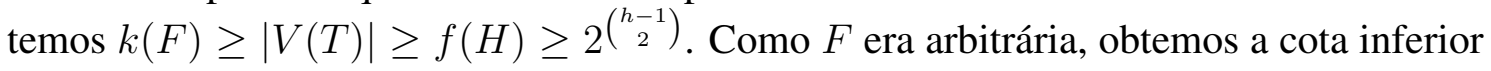
desejada.

Teorema 2.6. Seja $P_{d}$ o caminho com d arestas. Então $m_{F}\left(K_{1,3}, P_{d}\right)=d^{(1 / 2+o(1)) d}$.

A prova do teorema acima será omitida por motivos de espaço. Ela é análoga a do Teorema 2.5, substituindo a aplicação do Lema 2.4 pela desigualdade $f\left(P_{d}\right) \geq \sqrt{(d !)}=$ $d^{(1 / 2+o(1)) d}$. O seguinte corolário segue diretamente do Teorema 2.6.

Corolário 2.7. Para todo grafo $G$ de diâmetro $d$, vale que $m_{F}\left(K_{1,3}, G\right) \geq \sqrt{(d !)}$.

\section{Referências}

Alon, N., Jiang, T., Miller, Z., and Pritikin, D. (2003). Properly colored subgraphs and rainbow subgraphs in edge-colorings with local constraints. Random Structures \& Algorithms, 23(4):409-433.

Collares, M., Kohayakawa, Y., Moreira, C. G., and Mota, G. O. (2021). Constrained colourings of random graphs. Procedia Computer Science. To appear.

Gyárfás, A., Lehel, J., and Schelp, R. H. (2007). Finding a monochromatic subgraph or a rainbow path. Journal of Graph Theory, 54(1):1-12.

Jamison, R. E., Jiang, T., and Ling, A. C. (2003). Constrained ramsey numbers of graphs. Journal of Graph Theory, 42(1):1-16.

Loh, P.-S. and Sudakov, B. (2009). Constrained ramsey numbers. Combinatorics, Probability and Computing, 18(1-2):247-258. 